\title{
Los conocimientos tecnológicos, pedagógicos y de contenidos del profesorado universitario andaluz sobre las TIC. Análisis desde el modelo TPACK
}

\author{
The technological, pedagogical and content knowledge of Andalusian \\ university professors on ICT. Analysis from the TPACK model
}

\author{
D María Jesús Jiménez Sabino \\ Departamento de Didáctica y Organización Educativa, Universidad de Sevilla, España \\ marjimsab@alum.us.es \\ (iD) Julio Cabero Almenara \\ Departamento de Didáctica y Organización Educativa, Universidad de Sevilla, España \\ cabero@us.es
}

\section{RESUMEN}

El modelo TPACK es uno de los modelos de diagnóstico y formación del profesorado que mayor auge ha alcanzado en los últimos tiempos por su interés en los contenidos disciplinares, pedagógicos y tecnológicos. La literatura científica resalta que implementar este modelo en la enseñanza no solo ayuda a mejorar su calidad, sino que también permite determinar cuáles son los conocimientos del profesorado y diseñar estrategias de capacitación docente. Tomando como base estas consideraciones, los objetivos planteados en el estudio han sido tres: 1) conocer si el índice de fiabilidad del instrumento sobre el TPACK era similar al de otras investigaciones; 2) analizar los niveles de conocimientos de los participantes; 3) conocer si existían diferencias significativas entre los profesores según su Universidad. Por esta razón, hemos optado por una metodología cuantitativa con un diseño descriptivo y transversal. La muestra está formada por 396 profesores de cuatro Facultades públicas de Ciencias de la Educación de Andalucía occidental. Los resultados reflejan altos conocimientos disciplinares en comparación con las bajas puntuaciones alcanzadas en los dominios con presencia tecnológica. También se evidencia que el alfa de Cronbach es similar al de otras investigaciones y que no existen diferencias significativas en los conocimientos docentes según la Universidad. Como conclusiones relevantes, destacamos la homogeneidad en los resultados sobre los conocimientos de los participantes y la utilidad del modelo para la formación del profesorado.

PALABRAS CLAVE conocimiento del contenido pedagógico, formación docente, universidad, tecnología de la información, tecnología educativa.

\section{ABSTRACT}

The TPACK model is one of the most popular diagnose and teacher training models in recent times due to its interest in disciplinary, pedagogical and technological content. Scientific literature research that implements this model in teaching, not only helps to improve its quality but also allows us to determine what the teachers' allowance is and to design teacher 
training strategies. Based on these considerations, the objectives set out in the study were three: 1) to know if the reliability level of the instrument on the TPACK was similar to other investigations; 2 ) to analyze the amount of knowledge of the participants; 3) to know if there were significant differences between professors according to their University. For this reason, we have opted for a quantitative methodology with a descriptive and cross-sectional design. The sample is made up of 396 professors from four public Faculties of Education Sciences of western Andalusia. The results reflect high disciplinary knowledge in comparison with the low scores achieved in the domains with technological presence. It is also evident that Cronbach's alpha is similar to other investigations and that there are no significant differences in teaching knowledge according to the University. As relevant conclusions, we highlight the homogeneity in the results on the knowledge of the participants and the usefulness of the model for teacher training.

KEYWORDS pedagogical content knowledge, teacher education, universities, information technology, educational technology.

\section{INTRODUCCIÓN}

La sociedad del conocimiento ha posicionado a las tecnologías como una de las variables críticas en la enseñanza, y más aún en los momentos de pandemia que estamos viviendo. Las investigaciones evidencian en sus resultados que la tecnología suele emplearse como medio de información, en lugar de una herramienta pedagógica que potencie los niveles cognitivos (Ramma et al., 2018). Las tecnologías de la información y comunicación (TIC) "son consideradas prioritarias, no solo para alcanzar y contribuir a la alfabetización digital, sino también para apoyar el aprendizaje permanente a lo largo de toda la vida" (Herrero, 2014, p. 175). Son muchos los que afirman que su inclusión es tenue y con escaso impacto en los aprendizajes de los alumnos (Cuban, 2004; González et al., 2018; Howley et al., 2011; Tirado, \& Aguaded, 2014;). Por esta razón, es importante que la profesión docente sepa utilizar las tecnologías con fines pedagógicos (Oliver, 2012; Price, \& Kirkwood, 2014; Selwyn, 2014), saber qué tecnologías existen, cuáles son sus funcionamientos (Decoito, \& Richardson, 2018) y cómo llevarlas a la práctica (Livingstone, 2012). Ante los desafíos emergentes de la sociedad actual, la adquisición de competencias docentes es un tema que ha vuelto a tomar especial relevancia en los sistemas educativos (Infante et al., 2021; Loreli et al., 2019; Recio et al., 2020; Ruiz et al., 2020; Solís, \& Jara, 2019), tomando especial énfasis la competencia digital docente (Cabero, \& Palacios, 2020).

Para la formación docente se han formulado diferentes propuestas y modelos (Cabero, \& Martínez, 2019; García, \& Gutiérrez, 2020;), siendo el denominado modelo TPACK (Technological Pedagogical And Content Knowledge) de los que mayor significación ha obtenido con el paso de los años (Cabero et al., 2019). Mishra y Koehler (2006) afirman que "la buena enseñanza con tecnología requiere comprender las complejas y dinámicas interrelaciones entre la didáctica, el contenido y la tecnología en conjunto para desarrollar contextos específicos, estrategias y representaciones" (Koehler et al., 2015, en Flores et al., 2018, p. 122).

El TPACK asienta sus bases en los análisis de Lee Shulman (1986) sobre el conocimiento didáctico del contenido, entendido "como la mezcla de contenido y pedagogía en una comprensión de cómo temas, problemas o cuestiones particulares se organizan, representan y se adaptan a los diversos intereses y habilidades de los estudiantes" (Shulman, 1986, en Mailizar, \& Fan, 2020, p. 2). Shulman reconoció el valor del razonamiento pedagógico y creó categorías de conocimientos para el docente. Según Escudero et al., (2018), son ocho: 
1. Conocimiento de los contenidos disciplinares: comprende las áreas del conocimiento disciplinar que se incluyen en los currículos.

2. Conocimiento pedagógico general: incluye los conocimientos que trascienden a la enseñanza y que todo profesor tiene que conocer y aprender.

3. Conocimiento curricular: engloba programas, materias, recursos y herramientas para la enseñanza.

4. Conocimiento pedagógico/didáctico de los contenidos: hace referencia a las transformaciones del contenido disciplinar para preparar la enseñanza a través de actividades, recursos y decisiones que ayuden a la consecución del aprendizaje.

5. Conocimiento de los estudiantes: son los saberes previos, los estilos de aprendizajes y las características del alumnado.

6. Conocimiento de los contextos educativos: engloba el trabajo en grupo, el gobierno, las finanzas educativas, las comunidades y la cultura de los centros.

7. Conocimiento de las finalidades, valores educativos, bases filosóficas e históricas: formado por la justicia social y la equidad.

8. Razonamiento pedagógico: cómo se interpretan los contenidos, cómo se desarrollan los materiales, la preparación didáctica, la enseñanza, la reflexión y la creación de nuevos escenarios de aprendizajes (tanto individuales como grupales).

Mishra y Koehler (2006) toman las categorías de Shulman y diseñan un modelo teórico y formativo a partir de los diferentes conocimientos necesarios para introducir las TIC. Unwin (2007) agrega que lo llamativo de este modelo son las interrelaciones del conocimiento pedagógico y de contenido con el conocimiento tecnológico (ver Figura 1).

FIGURA 1. Interacciones de conocimientos del modelo TPACK (tpack.org)

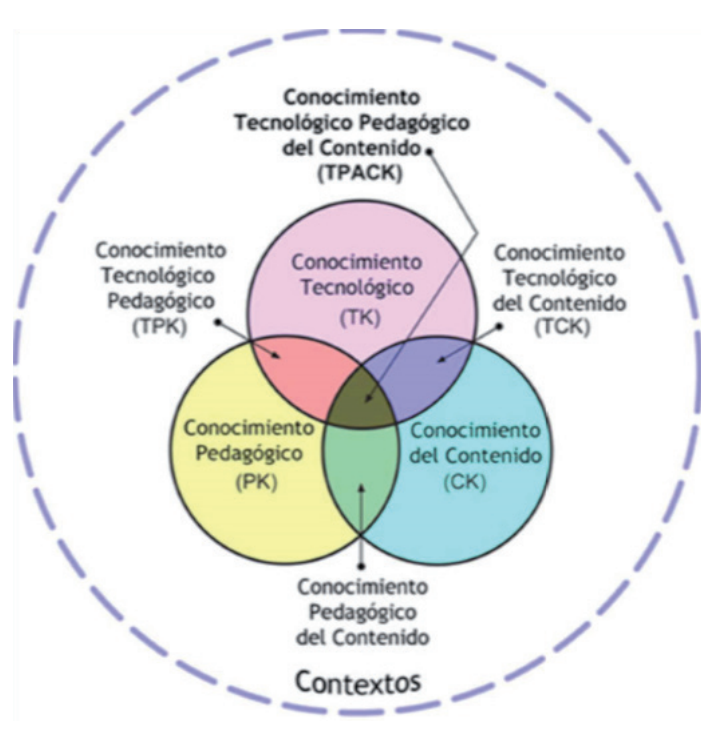

De acuerdo con McKenney y Voogt (2017), el modelo no obliga a que el profesor sea experto en el TPACK, pero sí que sepa dominar sus distintos conocimientos (Ramma et al., 2018): los principales (conocimiento tecnológico, conocimiento pedagógico y conocimiento del contenido) y sus interacciones (conocimiento del contenido pedagógico, conocimiento tecnológico pedagógico, conocimiento del contenido tecnológico y, por último, conocimiento tecnológico, pedagógico y de contenido). Roig et al. (2015), explican en qué consisten los conocimientos principales del TPACK:

- Conocimiento tecnológico o Technological Knowledge (TK): conocimiento sobre las creencias en tecnología, herramientas, aplicaciones, o recursos. 
- Conocimiento pedagógico o Pedagogical Knowledge (PK): conocimiento sobre las metodologías y los procesos de enseñanza.

- Conocimiento del contenido o Content Knowledge (CK): conocimiento de la materia que debe ser aprendida por el alumnado.

El resto de conocimientos del modelo se basan en los siguientes aspectos (Janssen et al., 2019; Samperio, \& Barrigán, 2018):

- Conocimiento del contenido pedagógico o Pedagogical Content Knowledge (PCK): conocimiento del sujeto, las actividades y las acciones sobre un tema concreto. Obliga a usar de formada adecuada los recursos pedagógicos para facilitar el aprendizaje de los alumnos.

- Conocimiento del contenido tecnológico o Technological Content Knowledge (TCK): conocimiento que incluye saber crear representaciones sobre un tema mediante las TIC.

- Conocimiento tecnológico pedagógico o Technological Pedagogical Knowledge (TPK): Rodríguez et al. (2019), entienden esta dimensión como las estrategias pedagógicas que son utilizadas cuando se usa la tecnología como puede ser elegir herramientas para una tarea, elegir herramientas según la eficiencia de la tarea o cuando se ponen en marcha elementos pedagógicos mientras se trabaja con TIC.

- Conocimiento tecnológico pedagógico de contenido o en inglés Technological Pedagogical And Content Knowledge (TPACK): conocimiento didáctico que tiene el profesorado para incorporar las TIC en cualquier área del conocimiento.

Según Cox (2008, en Phillips, 2016), el contexto hace que el modelo TPACK sea único, ubicado en un tiempo y lugar, idiosincrásico, adaptativo, específico y diferente para cada docente, de ahí la dificultad que genera su medición. Phillips (2016) critica la escasez de estudios acerca de su integración en los lugares de trabajo de los profesores en servicio y la complejidad de medir los niveles de TPACK por su carácter generalista (Özgen, \& Narl, 2020). Por ello, el diagrama del TPACK ha modificado recientemente su imagen canónica y ha convertido el contexto en un dominio de conocimiento (Mishra, 2019). Este nuevo dominio denominado conocimiento contextual o conteXtual knowlege (XK), emplea la X en su denominación al considerar el contexto como una variable que trata sobre el conocimiento que tiene el docente acerca de las tecnologías disponibles y las políticas educativas (Mishra, 2019).

A pesar de las críticas, el TPACK es considerado uno de los modelos más importantes por el énfasis que otorga al componente didáctico en su relación con los elementos tecnológicos e instrumentales (Barac et al., 2017; Cabero, 2014b;). Además, ayuda a diseñar y evaluar cursos de desarrollo profesional docente, conocer los conocimientos del profesorado en diversas áreas de la enseñanza y aumentar la calidad de la enseñanza (Agustin et al., 2019; Atiquil et al., 2019; Bulut y Isiksal, 2019; Deng et al. 2017; Djiwandono, 2019; Gyaase et al., 2019; Jang, \& Chang, 2016; Kaplon-Schilis, \& Lyublinskaya, 2019; Koh, 2018; Ying et al., 2016).

La significación del modelo ha tenido como consecuencia que se haya extendido su investigación, siendo algunos resultados los que se presentan a continuación:

- Atun y Usta (2019): la formación en TPACK genera mejores resultados en los aprendizajes.

- Beriy Sharma (2019): se han hallado diferencias en los niveles de TPACK según el género, la universidad y el tipo de universidad. 
- Cabero et al. (2017): existen altas autoevaluaciones de los conocimientos tecnológicos, pedagógicos y disciplinares por separado, pero bajos cuando deben considerarse conjuntamente.

- Horzum (2013): los niveles de TPACK aumentan en los profesores al concluir cursos sobre TIC y TPACK.

- Jang y Tsai (2012): el modelo ha demostrado resultados eficaces en los cursos de matemáticas y ciencias a partir del uso de las TIC.

- Ladrón et al. (2019): la puntuación del TPACK de un docente mejora cuando ha revisado acciones formativas centradas en tecnología.

- Redmond y Peled (2018): el TPACK es eficaz en los cursos de integración TIC para profesores antes del servicio.

- Takacs et al. (2015): la tecnología aporta resultados favorables en la alfabetización temprana, siempre y cuando el profesorado esté formado en el manejo y uso de las aplicaciones que vaya a necesitar.

- Valtonen et al. (2019): los profesores adquieren durante la docencia el conocimiento pedagógico del contenido (PCK). Después se hace difícil la incorporación del TPACK.

\section{MATERIAL Y MÉTODO}

\subsection{Objetivos de la investigación}

Los objetivos que hemos perseguido en el estudio han sido los que presentamos a continuación:

1. Conocer si el índice de fiabilidad del instrumento utilizado para el diagnóstico del TPACK de los profesores es similar al alcanzado en otras investigaciones.

2. Analizar las puntuaciones obtenidas por los profesores en los diferentes tipos de conocimientos según el modelo.

3. Conocer si existen diferencias significativas en las puntuaciones alcanzadas en el TPACK entre los profesores de las diferentes Universidades.

\subsection{Fases de la investigación}

En la tabla 1 detallamos las fases que hemos seguido durante la elaboración del estudio:

TABLA 1. Fases del proceso de investigación

\begin{tabular}{ccccccc}
\hline \multicolumn{7}{c}{ FASES DESARROLLADAS EN LA INVESTIGACIÓN } \\
\hline FASE I & FASE II & FASE III & FASE IV & FASE V & FASE VI & FASE VII \\
\hline $\begin{array}{c}\text { Identificación } \\
\text { del problema de } \\
\text { investigación }\end{array}$ & $\begin{array}{c}\text { Elección del } \\
\text { diseño de investi- } \\
\text { gación }\end{array}$ & $\begin{array}{c}\text { Elección de los } \\
\text { instrumentos de } \\
\text { recogida de datos }\end{array}$ & $\begin{array}{c}\text { Selección de la } \\
\text { muestra }\end{array}$ & $\begin{array}{c}\text { Recogida de los } \\
\text { datos facilitados } \\
\text { por el instrumento } \\
\text { utilizado }\end{array}$ & Análisis de & Cos datos \\
\hline
\end{tabular}




\subsection{La muestra de la investigación}

La muestra está formada por profesores de cuatro Facultades públicas de Ciencias de la Educación de Andalucía occidental: Cádiz, Córdoba, Huelva y Sevilla.

Para obtener la muestra averiguamos la población docente de todos los departamentos con sede interna de las cuatro Facultades alcanzando los siguientes valores:

- Cádiz: 198 profesores (100 hombres y 98 mujeres).

- Córdoba: 139 profesores (58 hombres y 81 mujeres).

- Huelva: 172 profesores (94 hombres y 78 mujeres).

- Sevilla: 258 profesores (118 hombres y 140 mujeres).

La muestra utilizada es del tipo no probabilística y del tipo de conveniencia o causal (Alaminos, 2006; Sabariego, 2012), que se basa en la facilidad de acceso del investigador a los sujetos que participan en el estudio.

Las fases de acceso a la población han sido dos: la primera en febrero de 2019 y la segunda retomada en noviembre de 2019 y finalizada en febrero de 2020. El contacto fue por correo electrónico, donde explicábamos quiénes éramos, cuál era nuestra Facultad y departamento y en qué consistía nuestra investigación. Explicamos que debían acceder a un cuestionario on-line realizado con Google Forms y que las respuestas iban a ser anónimas y tratadas para fines académicos.

La fase final nos permitió alcanzar un total de 396 respuestas al cuestionario. Los 396 profesores constituyen la muestra final. La tabla 2 presenta la población y muestra aceptante por Universidad y género.

En la tabla 3 presentamos la categoría profesional del profesorado que cumplimentó el cuestionario.

TABLA 2. Población y muestra de las Facultades de Cádiz, Córdoba, Huelva y Sevilla

\begin{tabular}{cccc}
\hline UNIVERSIDAD & GÉNERO & POBLACIÓN & MUESTRA \\
\hline \multirow{2}{*}{ Cádiz } & Hombres & 100 & 46 \\
\cline { 2 - 4 } & Mujeres & 98 & 54 \\
\hline Córdoba & Hombres & 58 & 24 \\
\cline { 2 - 4 } & Mujeres & 81 & 41 \\
\hline Huelva & Hombres & 94 & 42 \\
\cline { 2 - 4 } & Mujeres & 78 & 33 \\
\hline Sevilla & Hombres & 118 & 77 \\
\cline { 2 - 4 } & Mujeres & 140 & 79 \\
\hline
\end{tabular}

TABLA 3. Frecuencia y porcentaje de profesores en función de su categoría profesional

\begin{tabular}{ccc}
\hline $\begin{array}{c}\text { CATEGORÍA } \\
\text { PROFESIONAL }\end{array}$ & FRECUENCIA & PORCENTAJE \\
\hline $\begin{array}{c}\text { Contratado } \\
\text { Predoctoral }\end{array}$ & 14 & $3.5 \%$ \\
\hline Ayudante Doctor & 71 & $17.9 \%$ \\
\hline Contratado Doctor & 46 & $11.6 \%$ \\
\hline Profesor Titular & 119 & $30.1 \%$ \\
\hline $\begin{array}{c}\text { Catedrático } \\
\text { Universidad }\end{array}$ & 36 & $9.1 \%$ \\
\hline Profesor Asociado & 36 & $9.1 \%$ \\
\hline Sustituto Interino & 62 & $15.7 \%$ \\
\hline Otras categorías & 12 & $3.0 \%$ \\
\hline
\end{tabular}




\subsection{Instrumento de recogida de información}

Para este trabajo hemos utilizado la versión castellana de Cabero (2014a) del cuestionario de Schmidt et al. (2009), adaptado a las características de la muestra. Nuestro cuestionario está formado por 28 ítems agrupados en las siete dimensiones correspondientes al modelo TPACK:

- Conocimiento tecnológico (TK): 7 ítems.

- Conocimiento del contenido (CK): 2 ítems.

- Conocimiento pedagógico (PK): 7 ítems.

- Conocimiento pedagógico del contenido (PCK): 1 ítem.

- Conocimiento tecnológico del contenido (TCK): 1 ítem.

- Conocimiento tecnológico pedagógico (TPK): 5 ítems.

- Conocimiento tecnológico pedagógico del contenido (TPACK): 5 ítems.

Las respuestas del instrumento tienen una construcción tipo Likert: $\mathrm{MD}=$ muy en desacuerdo, $\mathrm{D}=$ en desacuerdo; N= ni de acuerdo ni en desacuerdo; $A=$ de acuerdo; y MA= muy de acuerdo.

Antes de la administración del cuestionario, calculamos su índice de fiabilidad mediante el coeficiente de consistencia interna alfa de Cronbach. Según Mateo (2004), los valores comprendidos en el intervalo 0.8 y 1 son considerados muy altos. En nuestro instrumento podemos observar el alto índice de fiabilidad alcanzado, tanto de forma global como en las diferentes dimensiones que lo conforman en comparación con los de otras investigaciones (ver Tabla 4).

TABLA 4. Índices de fiabilidad en las dimensiones del modelo TPACK

\begin{tabular}{|c|c|c|c|}
\hline $\begin{array}{l}\text { DIMENSIONES DEL } \\
\text { MODELO TPACK }\end{array}$ & $\begin{array}{l}\text { ÍNDICE DE FIABILIDAD DE } \\
\text { SCHMIDT ET AL. (2009) }\end{array}$ & $\begin{array}{c}\text { ÍNDICE DE FIABI- } \\
\text { LIDAD DE CABERO } \\
(2014 \mathrm{a})\end{array}$ & $\begin{array}{c}\text { ÍNDICE DE FIABILIDAD } \\
\text { DE NUESTRA INVESTIGACIÓN }\end{array}$ \\
\hline $\begin{array}{c}\text { Dimensión del conocimiento } \\
\text { tecnológico (TK) }\end{array}$ & 0.82 & 0.906 & 0.862 \\
\hline $\begin{array}{c}\text { Dimensión del conocimiento } \\
\text { del contenido (CK) }\end{array}$ & $\begin{array}{c}0.85 \text { (Matemáticas) } \\
0.84 \text { (Estudios sociales) } \\
0.82 \text { (Ciencia) } \\
0.75 \text { (Literatura) }\end{array}$ & 0.885 & 0.867 \\
\hline $\begin{array}{c}\text { Dimensión del conocimiento } \\
\text { pedagógico }(\mathrm{PK})\end{array}$ & 0.84 & 0.951 & 0.862 \\
\hline $\begin{array}{c}\text { Dimensión del conocimiento } \\
\text { pedagógico del contenido (PCK) }\end{array}$ & 0.85 & 0.787 & 0.869 \\
\hline $\begin{array}{l}\text { Dimensión del conocimiento } \\
\text { tecnológico del contenido (TCK) }\end{array}$ & 0.80 & 0.834 & 0.840 \\
\hline $\begin{array}{l}\text { Dimensión del conocimiento } \\
\text { tecnológico pedagógico (TPK) }\end{array}$ & 0.86 & 0.912 & 0.828 \\
\hline $\begin{array}{l}\text { Dimensión del conocimiento } \\
\text { tecnológico, pedagógico del } \\
\text { contenido (TPACK) }\end{array}$ & 0.92 & 0.899 & 0.832 \\
\hline
\end{tabular}




\section{RESULTADOS}

En primer lugar, presentamos las puntuaciones medias y desviaciones típicas alcanzadas por el profesorado en cada uno de los ítems. En la tabla 5 se puede observar la uniformidad de los valores de las medias. Las medias alcanzadas, con puntuaciones superiores a 3.5, sugieren que el profesorado está de acuerdo con los postulados indicados. Al mismo tiempo, los altos valores alcanzados en las desviaciones típicas sugieren cierta dispersión en las contestaciones.

TABLA 5. Medias y desviaciones típicas para los ítems del cuestionario

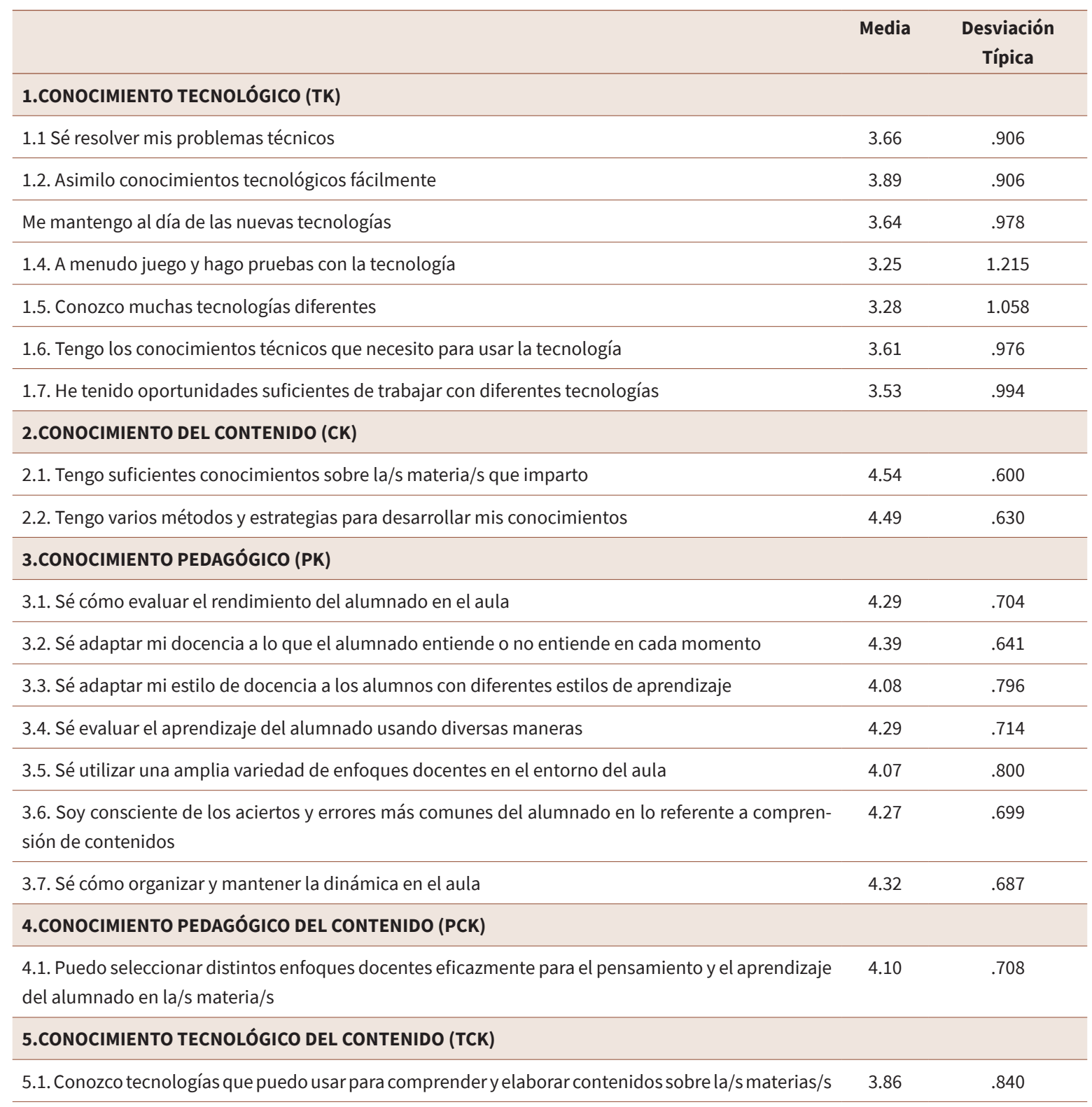




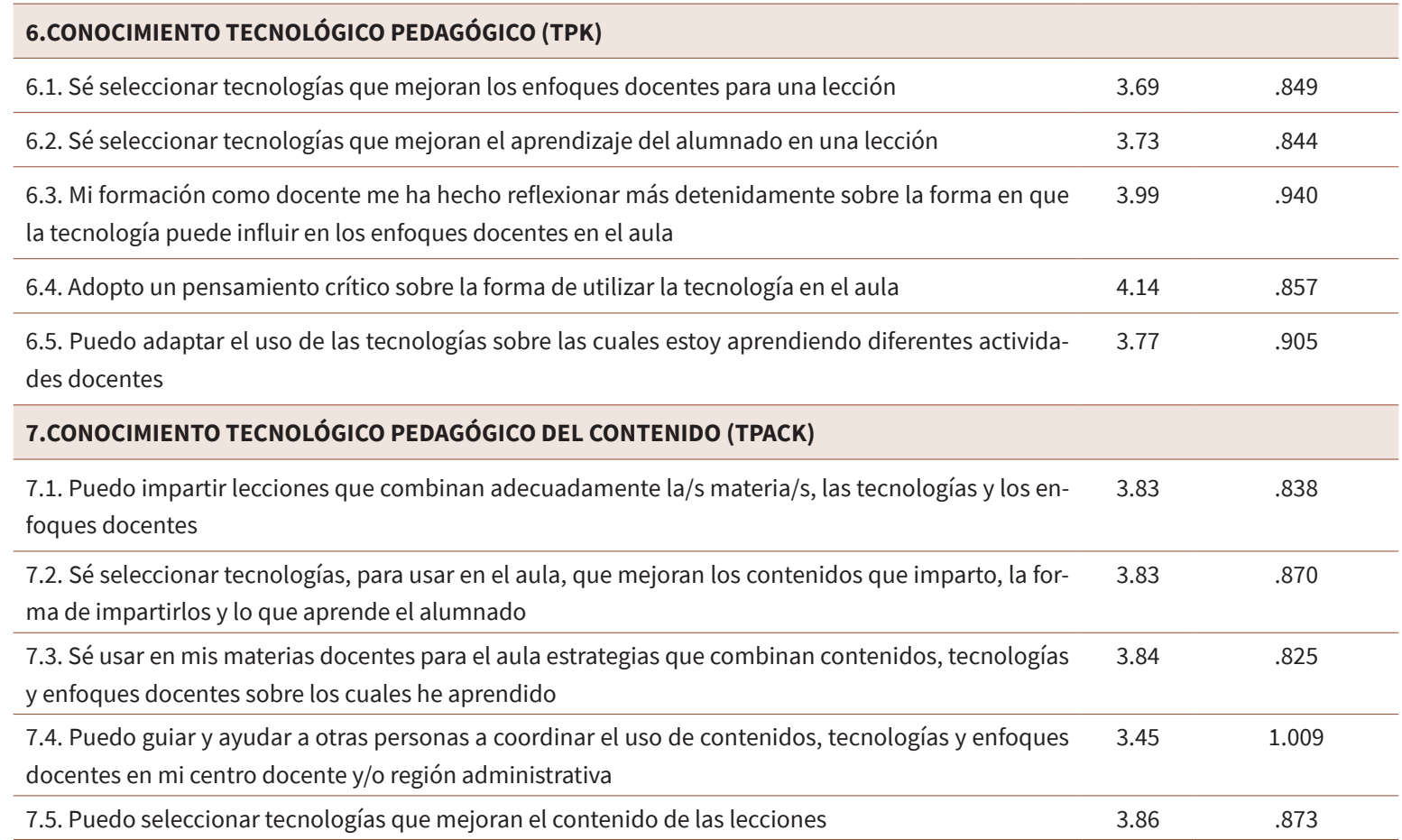

A continuación, presentamos las medias alcanzadas en cada una de las dimensiones que conforman el TPACK en la tabla 6. Las medias superan la puntuación 4 en las dimensiones sobre el conocimiento del contenido (CK), el conocimiento pedagógico (PK) y el conocimiento pedagógico del contenido (PCK). Las desviaciones típicas sugieren de nuevo dispersión.

TABLA 6. Medias y desviaciones típicas para las dimensiones del cuestionario

\begin{tabular}{llc}
\hline DIMENSIONES DEL CUESTIONARIO TPACK & Media & $\begin{array}{c}\text { Desviación } \\
\text { Típica }\end{array}$ \\
\hline CONOCIMIENTO TECNOLÓGICO (TK) & 3.5519 & .81628 \\
\hline CONOCIMIENTO DEL CONTENIDO (CK) & 4.5164 & .55494 \\
\hline CONOCIMIENTO PEDAGÓGICO (PK) & 4.2453 & .56132 \\
\hline CONOCIMIENTO PEDAGÓGICO DEL CONTENIDO (PCK) & 4.1010 & .70793 \\
\hline CONOCIMIENTO TECNOLÓGICO DEL CONTENIDO (TCK) & 3.8561 & .84013 \\
\hline CONOCIMIENTO TECNOLÓGICO PEDAGÓGICO (TPK) & 3.8662 & .72731 \\
\hline CONOCIMIENTO TECNOLÓGICO PEDAGÓGICO DEL CONTENIDO (TPACK) & 3.7626 & .77310 \\
\hline
\end{tabular}

Como señalamos, uno de los objetivos era analizar la existencia de diferencias significativas entre las Universidades participantes. Para ello, obtuvimos los valores medios ordenados, de mayor a menor (siendo 1 el valor más alto y 4 el más bajo), alcanzados por las Universidades para cada dimensión del cuestionario (ver Tabla 7). 
TABLA 7. Ordenación según la puntuación obtenida en las diferentes dimensiones por Universidad

\begin{tabular}{lcccc}
\hline & \multicolumn{3}{c}{ UNIVERSIDAD } \\
\cline { 2 - 4 } & Cádiz & Córdoba & Huelva & Sevilla \\
\hline CONOCIMIENTO TECNOLÓGICO (TK) & 2 & 1 & 4 & 3 \\
\hline CONOCIMIENTO DEL CONTENIDO (CK) & 3 & 2 & 4 & 1 \\
\hline CONOCIMIENTO PEDAGÓGICO (PK) & 3 & 4 & 1 & 2 \\
\hline CONOCIMIENTO PEDAGÓGICO DEL CONTENIDO (PCK) & 3 & 4 & 1 & 2 \\
\hline CONOCIMIENTO TECNOLÓGICO DEL CONTENIDO (TCK) & 4 & 2 & 3 & 1 \\
\hline CONOCIMIENTO TECNOLÓGICO PEDAGÓGICO (TPK) & 4 & 2 & 3 & 1 \\
\hline $\begin{array}{l}\text { CONOCIMIENTO TECNOLÓGICO PEDAGÓGICO DEL } \\
\text { CONTENIDO (TPACK) }\end{array}$ & 4 & 3 & 2 & 1 \\
\hline
\end{tabular}

Posteriormente, aplicamos el estadístico no paramétrico de Kruskal-Wallis para después formular las siguientes hipótesis:

- H0 (hipótesis nula): no existen diferencias significativas entre las dimensiones del cuestionario y la Universidad de los profesores.

- H1 (hipótesis alternativa): sí existen diferencias significativas entre las dimensiones del cuestionario y la Universidad de los profesores.

Al contar con un nivel de significación de 0.01 y un nivel de confianza del 99 \%, se aceptó la hipótesis nula para la globalidad del cuestionario (ver Tabla 8).

TABLA 8. Prueba de Kruskal-Wallis para las dimensiones del modelo según la Universidad

\begin{tabular}{lcc}
\hline & H de Kruskal-Wallis & Sig. asintótica \\
\hline CONOCIMIENTO TECNOLÓGICO (TK) & 1.897 & .594 \\
\hline CONOCIMIENTO DEL CONTENIDO (CK) & 2.217 & .529 \\
\hline CONOCIMIENTO PEDAGÓGICO (PK) & 3.831 & .280 \\
\hline CONOCIMIENTO PEDAGÓGICO DEL CONTENIDO (PCK) & 3.807 & .283 \\
\hline CONOCIMIENTO TECNOLÓGICO DEL CONTENIDO (TCK) & 3.570 & .312 \\
\hline CONOCIMIENTO TECNOLÓGICO PEDAGÓGICO (TPK) & 1.967 & .579 \\
\hline
\end{tabular}

\section{DISCUSIÓN Y CONCLUSIONES}

Las conclusiones de la presente investigación van en diferentes direcciones. Para su concreción se tomarán los objetivos planteados en el trabajo.

Por lo que se refiere a la fiabilidad del instrumento de diagnóstico es elevada, y se encuentra en consonancia con lo alcanzado en otros trabajos (Cabero, 2014a; Cabero et al., 2015; Cabero et al., 2017; Ladrón et al., 2020; Schmidt et al., 2009). 
Los resultados coinciden con los obtenidos por diferentes investigaciones que muestran una alta autoevaluación de los conocimientos pedagógicos y disciplinares (Cabero, 2014a; Ladrón et al., 2019; Roig, \& Flores, 2014). Al mismo tiempo, el modelo se presenta como un buen referente para la formación del profesorado (Leiva et al., 2018).

Los resultados muestran también cierta bajada en las puntuaciones cuando los diferentes tipos de conocimientos son considerados de forma conjunta. Resultado que coincide con otros trabajos (Cabero et al., 2015; Ladrón et al., 2020).

Finalmente, señalar que no se han encontrado diferencias significativas entre las diferentes Universidades, lo que sugiere, por una parte, la estabilidad en el colectivo de los conocimientos poseídos por los docentes según el modelo, y por otro cierta homogenización del profesorado en lo que se refiere a la formación y el conocimiento sobre las TIC.

\section{LIMITACIONES Y LÍNEAS FUTURAS DE INVESTIGACIÓN}

Las limitaciones que presenta el trabajo son de diversa índole. Unas se refieren al tipo de instrumento utilizado para analizar el TPACK, que tiene la característica de autoinforme. El cuestionario es un instrumento que no ofrece la realidad, sino la visión que el sujeto tiene de la misma. De ahí que sea necesario comenzar una línea de investigación dirigida a poder analizar el TPACK de los profesores a través de instrumentos más objetivos.

El tipo de muestreo, de conveniencia, tiene sus ventajas para el investigador, pero también sus limitaciones desde un punto de vista metodológico.

Y la investigación se ha centrado en las Universidades de Andalucía occidental, y en una facultad la de educación.

Las limitaciones comentadas sugieren al misFIGURA 2. Modelo TPeCS mo tiempo líneas futuras de investigación, que van en las siguientes direcciones: realizar la investigación con las Facultades de Andalucía oriental, y contrastar los resultados entre las diferentes Facultades. Extender su aplicación a otras Facultades de diferente tipología y analizar si se dan diferencias entre las mismas, por ejemplo, entre las ingenierías y las de ciencias de la educación.

Finalmente, consideramos conveniente que se comiencen a desarrollar trabajos con la propuesta realizada por Kali et al. (2019) denominada "Modelo TPeCS", que sugiere la contemplación de una nueva dimensión denominada espacio (ver Figura 2).

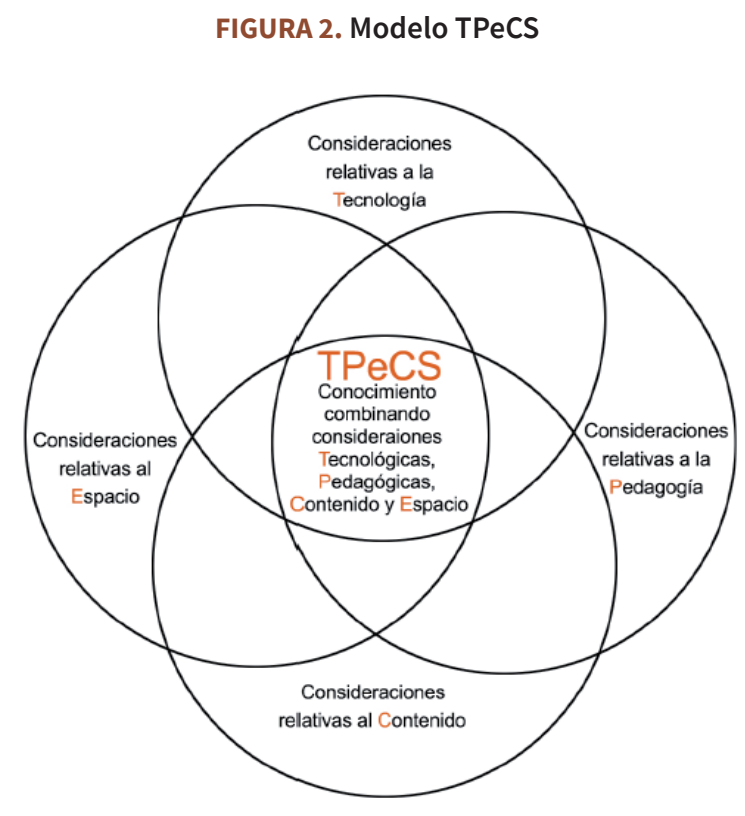




\section{REFERENCIAS}

Agustin, R. R., Liliasari, S., Sinaga, P., \& Rochintaniawati, D. (2019). Assessing pre-service science teachers' technological pedagogical content knowledge (TPACK) on kinematics, plant tissue and daily life material. Journal of Physics: Conference Series, 1157(2), 1-5. https://doi.org/10.1088/1742$6596 / 1157 / 2 / 022013$

Alaminos, A. (2006). El muestreo en la investigación social. En A. Alaminos y J.L. Castejón, Elaboración, análisis e interpretación de encuestas, cuestionarios y escalas de opinión (pp. 46-67). Alcoy.

Atiquil, A. Y. M., Mok, M. M. C., Gu, X., Spector, J. M., \& Hai-Leng, C. (2019). ICT in higher education: An exploration of practices in Malaysian universities. IEEE Access, 7, 16892-16908. https:// doi.org/10.1109/access.2019.2895879

Atun, H., \& Usta, E. (2019). The effects of programming education planned with TPACK framework on learning outcomes. Participatory Educational Research (PER), 6(2), 26-36. https://doi $\operatorname{org} / 10.17275 /$ per.19.10.6.2

Barac, K., Prestridge, S., \& Main, K. (2017). Stalled Innovation: Examining the technological, pedagogical and content Knowledge of Australian university educators. Australian Educational Computing, 32(1).

Beri, N., \& Sharma, L. (2019). A study on Technological and Content Knowledge among Teacher-Educators in Punjab Region. International Journal of Engineering and Advanced Technology (IJEAT), 8(5), 1306-1312. https://doi.org/10.35940/ijeat. $\underline{E} 1186.0585 C 19$

Bulut, A., \& Isiksal, M. (2019). Perceptions of pre-service elementary mathematics teachers on their technological pedagogical content knowledge (TPACK) regarding geometry. Journal of Computers in Mathematics and Science Teaching, 38(2), $153-176$

Cabero, J, Roig, R., \& Mengual, S. (2017). Conocimientos tecnológicos, pedagógicos y disciplinares de los futuros docentes según el modelo TPACK. Digital Education, (32), 73-84. https:// doi.org/10.1344/der.2017.32.73-84

Cabero, J. (2014a). La formación del profesorado en TIC: modelo TPACK. Plubidisa S.A.
Cabero, J. (2014b). Formación del profesorado universitario en TIC. Aplicación del método Delphi para la selección de los contenidos formativos. Educación XX1, 17(1), 111-132. https://doi.org/10.5944/educxx1.17.1.10707

Cabero, J., Arancibia, M.L., \& Del Prete, A. (2019). Conocimientos técnicos y didácticos del LMS de Moodle en la educación superior. Más allá del uso funcional. Revista de Nuevos Enfoques en la Investigación Educativa, 8(1), 25-33, http://dx.doi. org/10.7821/naer.2019.1.327

Cabero, J., Marín, V., \& Castaño, C. (2015). Validación de la aplicación del modelo TPACK para la formación del profesorado en TIC. @tic, Revista d'innovació educativa, (14), 13-22. http:// doi.org/10.7203/attic.14.4001

Cabero, J., \& Martínez, A. (2019). Las TIC y la formación inicial de los docentes. Modelos y competencias digitales. Profesorado. Revista de currículum y formación del profesorado, 23(3), 247268. https://doi.org/10.30827/profesorado.v23i3.9421

Cabero, J., \& Palacios, A. (2020). Marco Europeo de Competencia Digital Docente «DigCompEdu» y cuestionario «DigCompEdu Check-In». EDMETIC, Revista de Educación Mediática y TIC, 9(1), 213-234. https://doi.org/10.21071/edmetic.v9i1.12462

Cejas, R., Navío, A., \& Barroso, J. (2016). Las competencias del profesorado universitario desde el modelo TPACK (conocimiento tecnológico y pedagógico del contenido). Píxel-Bit. Revista De Medios Y Educación, (49), 105-119.

Cuban, L. (2004). The blackboard and the bottom line. Harvard University Press.

DeCoito, I., \& Richardson, T. (2018). Teachers and technology: Present practice and future directions. Contemporary Issues in Technology and Teacher Education, 180(2), 362-378.

Deng, F., Sing, C., So, H., Qian, Y., \& Chen, L. (2017). Examining the validity of the technological pedagogical content knowledge (TPACK) framework for preservice chemistry teachers. Australasian Journal of Educational Technology, 33(3), 1-14. https://doi.org/10.14742/ajet.3508

Djiwandono, P. I. (2019). How language teachers perceive information and communication technology. Indonesian Journal 
of Applied Linguistics, 8(3), 608-616. https://doi.org/10.17509/ ijal.v8i3.15260

Escudero, J. M., González, M. T., \& Rodríguez, M. J. (2018). Los contenidos de la formación continuada del profesorado: ¿Qué docentes se están formando? Educación XX1, 21(1), 157-180. https://doi.org/10.5944/educXX1.15807

Flórez, L. D., Ramírez, C. R., \& Ramírez, S. R. (2016). Las TIC como herramientas de inclusión social. 3C TIC, 5(1), 54-67. https:// doi.org/10.17993/3ctic.2016.51.54-67

García, Y., \& Gutiérrez, P. (2020). El rol docente en la sociedad digital. Digital Education Review, (38), 1-22. https://revistes. ub.edu/index.php/der/article/view/27102

González, N., Ramírez, A., \& Salcines, I. (2018). Competencia mediática y necesidades de alfabetización audiovisual de docentes y familias españolas. Educación XX1, 21(2), 301-321. https://doi.org/10.5944/educXX1.16384

Gyaase, P. O., Gyamfi, S. A., \& Kuranchie, A. (2019). Gauging the e-readiness for the integration of information and communication technology into pre-tertiary education in Ghana: An assessment of teachers' technological pedagogical content knowledge (TPACK). International Journal of Information \& Communication Technology Education, 15(2), 1-17. https:// doi.org/10.4018/ijicte.2019040101

Herrero, R.M. (2014). El papel de las TIC en el aula universitaria para la formación en competencias del alumno. Píxel-Bit. Revista de Medios y Educación, (45), 173-188. http://hdl.handle. net/11441/46215

Horzum, M. B. (2013). An investigation of the technological pedagogical content knowledge of pre-service teachers. Technology, Pedagogy and Education, 22(3), 303-317. https://doi.org/1 $\underline{0.1080 / 1475939 \times .2013 .795079}$

Howley, A., Wood, L., \& Hough, B. (2011). Rural elementary school teachers' technology integration. Journal of Research in Rural Education, 26(9), 1-13.

Infante, A., Infante, J., \& Gallardo, J. (2021). The acquisition of ICT skills at the university level: the case of the Faculty of Business Studies and Tourism of the University of Huelva. Píxel-Bit. Revista de Medios y Educación, (60), 29-58. https:// doi.org/10.12795/pixelbit.79471. https://doi.org/10.12795/ pixelbit.79471
Jang, S.Y., \& Chang, Y. (2016). Exploring the technological pedagogical and content knowledge (TPACK) of Taiwanese university physics instructors. Australasian Journal of Educational Technology, 32(1), 107-122. https://doi.org/10.14742/ajet.2289

Jang, S.Y., \& Tsai, M.F. (2012). Exploring the TPACK of Taiwanese elementary mathematics and science teachers with respect to use of interactive whiteboards. Computers \& Education, 59(2), 327-338. https://doi.org/10.1016/j.compedu.2012.023

Janssen, N., Knoef, M., \& Lazonder, W. (2019) Technological and pedagogical support for pre-service teachers' lesson planning. Technology, Pedagogy and Education, 28(1), 115-128. https://doi.org/10.1080/1475939X.2019.1569554

Kali, Y., Sagy, O., Benichou, M., Atias, O., \& Levin, R. (2019). Teaching expertise reconsidered: The Technology, Pedagogy, Content and Spaces (TPeCS) knowledge framework. British Journal of Educational Technology, 50(5), 2162-2177. https:// doi.org/10.1111 / bjet. 12847

Kaplon-Schilis, A., \& Lyublinskaya, I. (2019). Analysis of relationship between five domains of TPACK framework: TK, PK, CK math, CK science, and TPACK of pre-service special education teachers. Technology, Knowledge and Learning. Advance online publication. https://doi.org/10.1007/s10758-019-09404-x

Koh, J. H. L. (2018). TPACK design scaffolds for supporting teacher pedagogical change. Educational Technology Research and Development. Advance online publication. https://doi. org/10.1007/s11423-018-9627-5

Ladrón, L. (2020). Las TIC en la educación física actual: estudio del conocimiento tecnológico, pedagógico y disciplinar (TPACK) en el profesorado universitario de educación física en España. [Tesis doctoral no publicada]. Universidad de Sevilla.

Ladrón, L., Cabero, J., \& Almagro, B. (2019). El conocimiento tecnológico, pedagógico y disciplinar del profesorado de Educación Física. Retos, (36), 362-369. https://doi.org/10.47197/ retos.v36i36.68898

Leiva, J. P., Ugalde, L., \& Llorente, C. (2018). El modelo TPACK en la formación inicial de profesores: modelo Universidad de Playa Ancha (UPLA), Chile. Píxel-Bit. Revista De Medios Y Educación, (53), 165-177. https://doi.org/10.12795/pixelbit.2018. i53.11. 
Livingstone, S. (2012). Critical Reflections on the benefits of ICT in Education. Oxford Review of Education, 38(1), 9-24. https:// doi.org/10.1080/03054985.2011.577938

Loreli, A., Gamiz, V., \& Romero, M.A. (2019). Niveles de desarrollo de la competencia digital docente:una mirada a marcos recientes del ámbito internacional. Innoeduca, International Journal Of Technology And Educational Innovation, 5(2), 140150. https://doi.org/ 10.24310/innoeduca.2019.v5i2.5600.

Mailizar, M., \& Fan, L. (2020). Indonedian Teachers 'Knowledge of ICT and the Use of ICT in Secondary Mathematics Teaching. Journal of Mathematics, Scince and Technology Education, 16(1), 1-13. https://doi.org/10.29333/ejmste/110352

Mateo, J. (2004). La investigación ex-post-facto. En R. Bisquerra (coord.), Metodología de la investigación educativa (pp. 195230). La Muralla.

McKenney, S., \& Voogt, J. (2017). Expert views on TPACK for early literacy: Priorities for teacher education. Australasian Journal of Educational Technology, 33(5), 1-14. https://doi. org/10.14742/ajet.2502

Mishra, P. (2019). Considering Contextual Knowledge: The TPACK Diagram Gets an Upgrade. Journal of Digital Learning in Teacher Education, 35(2), 76-78. https://doi.org/10.1080/215329 74.2019 .1588611

Mishra, P., \& Koehler, J. (2006). Technological Pedagogical Content Knowledge: A new framework for teacher knowledge. Teachers College Record, 108(6), 1017-1054.

Oliver, M. (2012). Technology and Change in Academic Practice. En P. Trowler, M. Saunders \& V. Bamber (Eds.), Tribes and Territories in the 21st Century: Rethinking the Significance of Disciplines in Higher Education. International Studies in Higher Education: ERIC.

Özgen, K., \& Serkan, N. (2020). Intelligent Data Analysis of Interactions and Relationships among Technological Pedagogigacal Content Knowledge Constructs via Rough Set Analysis. Contemporary Educational Technology, 11(1), 77-98. https://doi. org/10.30935/cet.646769

Phillips, M. (2016). Processes of practice and identity shaping teachers' TPACK enactment in a community of practice. Educa- tion and Information Technologies, 22(4), 1771-1796. https:// doi.org/10.1007/s10639-016-9512-y

Price, L., \& Kirkwood, A. (2014). Using technology for teaching and learning in higher education: a critical review of the role of evidence in informing practice. Higher Education Research and Development, 33(3), 549-564. https://doi.org/10.1080/07 $\underline{294360.2013 .841643}$

Ramma, Y., Bholoa, A., Watts, M., \& Sylvain, P. (2018) Teaching and learning physics using technology: Making a case for the affective domain. Education Inquiry, 9(2), 210-236. https:// doi.org/10.1080/20004508.2017.1343606

Recio, F., Silva, J., \& Abricot, N. (2020). Análisis de la Competencia Digital en la Formación Inicial de estudiantes universitarios: Un estudio de meta-análisis en la Web of Science. Píxel-Bit. Revista De Medios Y Educación, (59), 125-146. https://doi. org/10.12795/pixelbit.77759.

Redmond, P., \& Peled, Y. (2018). Exploring TPACK among preservice teachers in Australia and Israel. British Journal of Educational Technology. Advance online publication. https://doi. org/10.1111/bjet.12707

Roig, R., Mengual, S., \& Quinto, P. (2015). Conocimientos tecnológicos, pedagógicos y disciplinares del profesorado de Primaria. Comunicar, 23(45), 151-159. https://doi.org/10.3916/ C45-2015-16

Roig, R., \& Flores, C. (2014). Conocimiento tecnológico, pedagógico y disciplinario del profesorado: el caso de un centro educativo inteligente. EDUTEC. Revista Electrónica de Tecnología Educativa, (47). https://doi.org/10.21556/edutec.2014.47.93

Ruiz, A., Medina, M., Pérez, E., \& Medina, A. (2020). University teachers' training: the Digital Competence. Píxel-Bit. Revista De Medios Y Educación, (58), 181-215. https://doi.org/10.12795/ pixelbit.74676.

Sabariego, M. (2012). El proceso de investigación (parte 2). En R. Bisquerra (coord.), Metodología de la investigación educativa (pp. 127-163). La Muralla.

Samperio, V.M., \& Barragán, J.F. (2018). Análisis de la percepción de docentes, usuarios de una plataforma educativa a través de los modelos TPACK, SAMR Y TAM3 en una institución de 
educación superior. Apertura, 10(1), 116-131. https://doi. org/10.32870/ap.v10n1.1162

Selwyn, N. (2014). Digital Technology and the Contemporary University: Degrees of Digitization. Routledge.

Shulman, L. (1986). Those who understand: Knowledge growth in teaching. Educational Researcher, 1(2), 4-14.

Solís de Ovando, J., \& Jara, V. (2019). Competencia digital de docentes de Ciencias de la Salud de una universidad chilena. Píxel-Bit. Revista De Medios Y Educación, (56), 193-211. https://doi.org/10.12795/pixelbit.2019.i56.10.

Takacs, Z. K., Swart, E. K., \& Bus, A. G. (2015). Benefits and pitfalls of interactive features in technology-enhanced storybooks. A meta-analysis. Review of Educational Research, 85(4), 698739. https://doi.org / $10.3102 / 0034654314566989$

Tirado, R., \& Aguaded, J.I. (2014). Influencias de las creencias del profesorado sobre el uso de la tecnología en el aula. Revista de Educación, (363), 230-255. https://doi.org/10.4438/1988592X-RE-2012-363-179

Valtonen, T., Sointu, E. Kukkonen, J., Mäkitalo, K., Häkkinen, P., Järvela, S., Näykki, P., Virtanen, A., Pöntinen, S., Kostiainen, E., \& Tondeur, J. (2019). Examining pre-service teachers' Technological Pedagogical Content Knowledge as evolving knowledge domains: Alongitudinal approach. Journal of Computer Assisted Learning, 35(4), 491-502. https://doi. org/10.1111/jcal.12353

Yi, Y., Ying, H., Hsin, W., \& Sung, C. (2016). Exploring the structure of TPACK with videoembedded and discipline-focused assessments. Computers \& Education, 104, 49-64. https://doi. org/10.1016/j.compedu.2016.106 